Introduction Inflammatory bowel disease (IBD) can have a significant impact on physical, psychological and social wellbeing. We aimed to survey the impact of IBD on our patients' lives and their perceptions of psychological support use and availability.

Methods Inflammatory bowel disease (IBD) can have a significant impact on physical, psychological and social wellbeing. We aimed to survey the impact of IBD on our patients' lives and their perceptions of psychological support use and availability.

Results 6 patients were excluded as they had not completed the questionnaire. 94 patients were included (43 male, 51 female, average age 42 years, range 17-76). 46 had a diagnosis of Crohn's disease, 41 ulcerative colitis and 7 indeterminate colitis. Average disease duration for these patients was 15 years (range 1-51 years). Over the past 6 months 20 had symptoms constantly, 16 often, 17 occasionally, 10 sometimes, 13 rarely and 18 never. The average SIBDO score was 48 (range 21-70). The average HAD score was 12.6 (range $0-33$ ). When separated into HAD A (anxiety) and HAD D (depression) scores were 8.1 (range $0-18$ ) and 4.8 (range $0-15$ ) on average respectively, a score of 8 to 10 for either subscale being suggestive of the presence of the respective state. They were also asked which services they had previously used as forms of support. Of the 86 patients who answered this portion of the questionnaire, $13(15 \%)$ said they had previously had counselling or psychological input, and $32(37 \%)$ said they would like counselling or psychological input in the future if it was available.

Conclusion Our survey suggests there may be a higher rate of anxiety in patients with IBD, and that over a third of our patients would like access to psychological and counselling services if they were available. Psychological support is important to patients with IBD and should be incorporated into their management.

Disclosure of Interest None Declared.

\section{PTH-101 THE BURDEN OF IRON DEFICIENCY ANAEMIA IN A TERTIARY IBD CENTRE POPULATION}

doi:10.1136/gutjnl-2013-304907.588

1."S Esmaily, 'S Hearnshaw, ' J Mansfield, 'R Speight, 'M Gunn. 'Department of Gastroenterology, Royal Victoria Infirmary, Newcastle Upon Tyne, UK

Introduction Anaemia is the most common extraintestinal manifestation of IBD, with iron deficiency being the most prevalent cause. The ECCO guidelines published in 2012 included the management of anaemia in UC. Previous studies have shown up to $1 / 3$ of the IBD population are anaemic. Anaemia may affect quality of life in patients. There remains uncertainty in the clinical benefit in treatment of mildly anaemic and iron deficient patients. The aim of the study was to identify the burden of iron deficiency anaemia in patients with IBD in a tertiary referral centre and whether we are treating this according to the ECCO guidelines.

Methods Consecutive patients with IBD reviewed in general gastroenterology and specialist IBD clinics during one month period extending from the 1st of December 2012 to the 1st of January 2013 were included. $\mathrm{Hb}, \mathrm{MCV}$, ferritin and transferrin saturations were recorded. Results were documented prospectively. Iron deficiency was diagnosed on the basis of recent ECCO guidelines. Anaemia was defined as $\mathrm{Hb}<12 \mathrm{~g} / \mathrm{dL}$ in females and $<13 \mathrm{~g} / \mathrm{dL}$ in males Treatment with oral or parenteral iron was also noted.

Results Out of 104 patients seen during this period, 21 (20.2\%) patients had iron deficiency out of which 11 (10.6\%) were anaemic $3 / 11(27.3 \%)$ patients with iron deficiency anaemia had a $\mathrm{Hb}$ of less than $10,2 / 3$ of which received parental iron. One received no treatment. Out of the remaining patients with milder iron deficiency anaemia ( $\mathrm{Hb}>10 \mathrm{~g} / \mathrm{dL}), 3$ had no treatment, 1 had IV only, 2 had oral only and 2 had both IV and oral. In the Iron deficient non anaemic group, $6 / 10$ had no treatment, $2 / 10$ had oral iron and $2 / 10$ had parental iron.
Conclusion Our study showed $20.2 \%$ of IBD patients attending over a one month period had iron deficiency anaemia or iron deficiency. Treatment of iron deficiency with and without anaemia is variable and could improve with adherence to a clear protocol. The question of whether the treatment of iron deficiency without significant anaemia in IBD improves fatigue and QOL requires further research.

Disclosure of Interest None Declared.

\section{PTH-102 PRELIMINARY EVIDENCE FOR A ROLE OF THE RENIN ANGIOTENSIN SYSTEM IN INTESTINAL FIBROSIS IN CROHN'S DISEASE USING ANGIOTENSIN RECEPTOR IMMUNOHISTOCHEMISTRY}

doi:10.1136/gutjnl-2013-304907.589

1,2, S Ray, ${ }^{2} \mathrm{~J}$ Soeda, ${ }^{1,2 \mathrm{~J}}$ Oben, ' $\mathrm{J}$ Sanderson. 'Gastroenterology, Guy's \& St Thomas' Hospitals; '2Institute of Liver \& Digestive Health, University College London, London, UK

Introduction Fibrosis is a major limitation to successful non-surgical treatment of Crohn's disease (CD) and is a cause of significant morbidity. The pathophysiological determinants of intestinal fibrosis in CD remain uncertain. The renin-angiotensin system (RAS) plays a critical role in blood pressure control and fluid and electrolyte homeostasis but has recently been implicated in fibrogenesis in multiple organ systems. Angiotensin II is the main active peptide in the RAS and evidence suggests it has important proliferative and profibrogenic roles acting through its AT1 receptor. Pharmacological blockade of the AT1 receptor has become a key element in the management of chronic cardiac and renal disorders and abrogates fibrosis. It also appears anti-fibrotic in chronic liver disease. Subsequent studies suggest that the AT2 receptor may act to oppose many of the proliferative and fibrogenic actions of AT1. Few studies have investigated a role for the RAS in intestinal fibrosis in CD.

Aim A preliminary study to use immunohistochemistry to determine AT1 and AT2 expression in resected ileum of patients with CD. Methods Immunohistochemistry for AT1 and AT2 receptors was performed on archival formalin fixed paraffin embedded ileum of patients who had undergone resection for stricturing $\mathrm{CD}(\mathrm{n}=6)$, histologically normal ileum in patients undergoing colectomy for ulcerative colitis (UC) $(n=4)$ or right hemicolectomy for colon cancer $(n=1)$. We used commercial antibodies for AT1(SC1173) and AT2(SC9040) and the secondary antibody (Vector ImmPRESS Anti-Rabbit Ig (peroxidase) Polymer Detection Kit) under optimised conditions.

Results AT1 and AT2 staining patterns differed quite clearly between $\mathrm{CD}$ resection samples and those from UC and cancer controls. Control and UC cases showed clear staining of the muscularis for AT1 but consistently negative staining of epithelial or lamina propria compartments, whilst AT2 stained only the epithelial layer. Conversely, each of the 6 CD cases showed clear AT1 staining of the epithelial layer as well as muscularis but negative staining for AT2 in all areas. Occasional myofibroblast-like cells were also specifically stained by AT1. RTPCR analysis of RNA from CCD18CO cells (intestinal myofibroblast cell line) confirmed AT1 expression and no AT2 expression.

Conclusion These preliminary findings demonstrate a distinct difference in angiotensin receptor expression pattern in CD and nonCD ileum. This suggests a possible alteration in the balance between AT1 and AT2 receptor expression in CD strictures contributing to fibrogenesis and certainly merits further investigation

Disclosure of Interest None Declared.

\section{PTH-103 CONTROLLING THE BALANCE OF IMMUNITY IN COLITIS: INVESTIGATING THE ROLES OF INTESTINAL MICROBIOTA AND DENDRITIC CELL MIGRATION}

doi:10.1136/gutjnl-2013-304907.590

1.*S Borg-Bartolo, ${ }^{2} \mathrm{~J}$ McLaughlin, ${ }^{3} \mathrm{~S}$ Cruickshank. 'Department of Immunology; ${ }^{2} S c h o o l$ of Translational Medicine, University of Manchester/NHS; ${ }^{3}$ Department of Immunology, University of Manchester, Manchester, UK 
Introduction The pathogenesis of Inflammatory Bowel Disease (IBD) is unclear which hinders effective targeted drug development. IBD and murine models of colitis are associated with the abnormal accumulation of activated dendritic cells (DCs) in the colonic epithelium. DCs play a critical role in promoting inflammatory responses and blockade of their activation prevents colitis development in mouse models. We now propose to address the mechanisms underlying the aberrant accumulation of DCs in the gut by focusing on microbial danger stimuli that drive activation and migration of DCs. Methods We analysed the expression of migration-associated markers on DCs from normal and colitic mice. Bone marrow derived DCs (BMDCs) from WT or Beta-2 Integrin ${ }^{-/}\left(\right.$ITGB2 $\left.^{-/}\right)$mice were cultured in vitro and their migration and activation analysed in response to control (Phosphate buffered saline - PBS), bacterial lipopolysaccharide (LPS), live Escherichia coli (EC), and live Bacteroides fragilis $(\mathrm{BF})$ in the presence and absence of the lamina propria extracellular matrix component fibronectin.

Results During colitis there was a marked increase in a population of CD103- ( $a_{\mathrm{E}}$ integrin) DCs. We were able to mirror these populations in vitro. DCs moved via random motion and their velocity after stimulation with LPS and EC, in the absence of fibronectin, was significantly decreased. In comparison, stimulation with BF significantly increased DC velocity ( $p<0.001$ for all). In the presence of fibronectin, there was no change in DC velocity. The track displacement length (the distance between the start and finishing point of a given cell migration track) was significantly decreased after EC stimulation and significantly increased after BF stimulation ( $p<0.01$ for both). Unstimulated ITGB2 $\%$ BMDC velocity and track displacement length were significantly increased in comparison to that of unstimulated WT BMDC ( $p<0.001$ for both). This was more marked in the absence of fibronectin.

Conclusion We have shown that in vitro WT DC cultures contain DCs with similar integrin-defined phenotypes to those found in colonic DCs in colitis. Differential bacterial stimulation causes opposing fibronectin-dependent effects on BMDC migratory behaviour whilst absence of ITGB2 significantly alters the migratory behaviour of BMDC. Our data implicates a complex relationship between specific components of gut microbiota, extracellular matrix, and migration and activation of DCs that could potentiate the aberrant accumulation of DCs in the colitic gut. If this complex relationship is further elucidated, it may be possible in the future to develop therapies that reduce colitis by controlling DC migration. Disclosure of Interest None Declared.

\section{PTH-104 THE ROUTINE MEASUREMENT OF THIOPURINE METABOLITE LEVELS RESULTS IN DOSE OPTIMISATION IN ONE THIRD OF IBD PATIENTS; RESULTS FROM A DISTRICT GENERAL HOSPITAL}

doi:10.1136/gutjnl-2013-304907.591

${ }^{1} \mathrm{H}$ M Dewhurst, ${ }^{2} \mathrm{H}$ E Johnson, ${ }^{3} \mathrm{~J}$ Begley, ${ }^{2} \mathrm{~S}$ A Weaver, ${ }^{2,}$ S D McLaughlin. ${ }^{1}$ Research; ${ }^{2}$ Gastroenterology; ${ }^{3}$ Clinical Biochemistry, Royal Bournemouth Hospital, Bournemouth, UK

Introduction Measuring azathioprine or mercaptopurine (AZA) metabolite levels 6-TGN and 6-MMPN allows identification of patients who are: 1 . Non compliant with their medication, 2. On a sub-optimal doe, 3. On a supra-therapeutic dose, 4. Are preferentially metabolising azathioprine to methylated metabolites (6-MMPN:6-TGN ratio > 11).

Our own and others published data demonstrate that measuring metabolite levels in patients failing azathioprine therapy followed by appropriate changes in dosing and/or the addition of allopurinol (with $75 \%$ dose reduction in AZA) can result in clinical remission in the majority of patients 1 . We report the outcome of the routine measurement of metabolite levels in patients treated with AZA who were in a clinical remission without side effects or abnormal liver function tests (LFTs).

Methods All patients underwent TPMT testing, azathioprine and mercaptopurine were initiated at doses of $2 \mathrm{mg} / \mathrm{kg}$ and $1 \mathrm{mg} / \mathrm{kg}$ respectively in those with wild-type TPMT with a $50 \%$ reduction in dose in TPMT heterozygotes. We searched the prospective database maintained by our biochemistry department for all patients who underwent metabolite level testing from September 2011 to November 2012, hospital case notes for these patients were reviewed. The indications, results of testing, changes in clinical management and patient outcomes were recorded.

Results 108 patients underwent metabolite testing, median length of follow-up since testing was 287.6 days (range 21-441), all were stable on AZA for $>4$ weeks with normal LFTs and in a clinical remission. 38 (35.2\%) had UC, 66 (61.1\%) CD, 52 (48.1\%) were male.

$17(15.7 \%)$ patients had a sub-theraputic 6-TGN, 10 (9.3\%) supra-theraputic 6-TGN level $(>800)$ all of whom had dose optimisation. $6(5.6 \%)$ patients were hypermethylators these were switched to allopurinol co-therapy with an appropriate reduction in AZA dose.

Conclusion In the present study the routine measurement of AZA metabolites resulted in a change in clinical management in $30.6 \%$ of patients.

Whilst unproven in prospective longitudinal studies logic suggests that the routine measurement of AZA metabolites in all patients commenced on thiopurines followed by appropriate dose optimisation (with or without allopurinol co-prescription) should reduce or prevent the development of drug side effects, abnormal LFTs and bone marrow suppression and reduce the risk of disease relapse. We recommend that AZA metabolite testing is performed in all patients 4-6 weeks after commencing AZA.

Disclosure of Interest None Declared.

\section{PTH-105 THE EFFECT OF INFLIXIMAB PRE-TREATED HUMAN BLOOD- ENRICHED DENDRITIC CELLS FROM PATIENTS WITH ACTIVE CROHN'S DISEASE AND HEALTHY CONTROLS ON SUBSEOUENT HUMAN T-LYMPHOCYTE PHENOTYPE AND CYTOKINE PRODUCTION IN VITRO}

doi:10.1136/gutjnl-2013-304907.592

1. S T C Peake, ${ }^{2} \mathrm{D}$ Bernardo, ${ }^{2} \mathrm{E}$ Mann, ${ }^{1} \mathrm{~J}$ Landy, ${ }^{2} \mathrm{H}$ Omar, ${ }^{2} \mathrm{~S}$ C Knight, ${ }^{1} \mathrm{~A}$ L Hart. ${ }^{1} / B D$ Unit, St Mark's Hospital; ${ }^{2} A P R G$, Imperial College, London, UK

Introduction Dendritic cells (DC) play a key role in discriminating between commensal microorganisms and potentially harmful pathogens. Expression of surface markers and cytokine production by DC at the time of antigen presentation control T-cell differentiation, cytokine profile \& homing properties imprinted on stimulated T-cells. This process defines the type of immune response that occurs and its anatomical location. In $\mathrm{CD}$, dysregulation of the immune response to gut microbiota and aberrant immune cell trafficking play a central role in disease pathogenesis. Infliximab (IFX) is an effective treatment for CD, but its mechanism of action is unclear. In this study, we investigated the effect of IFX pre-treated blood-enriched DC, isolated from patients with active CD and healthy controls (HC), on human T-cell proliferation, phenotype\&cytokine production

Methods Low density cells (LDC), enriched for DC, were obtained following Ficoll and Nycoprep gradient separation of fresh blood from patients with active ileocolonic CD (CDAI > 220) and HC. LDC were cultured $\left(0.5 \times 10^{6} \mathrm{cells} / \mathrm{ml}\right)$ with IFX $(1.10 \mu \mathrm{g} \& 100 \mu \mathrm{g} /$ $\mathrm{ml}$ \&basal) for $24 \mathrm{hr}$. T-cells were enriched from allogeneic $\mathrm{HC}$ blood and labelled with CFSE. LDC were added to T-cells in complete medium $(400,000$ cells $/ \mathrm{ml})$ at basal,1.2\&3\% concentrations and incubated for 5 days. Following incubation, T-cell proliferation, 